\title{
TTR
}

Traduction, terminologie, re?daction

\section{À propos d'une expérience de traduction : Désir sous les ormes d'Eugène O’Neill}

\section{Françoise Morvan}

Volume 7, numéro 2, 2e semestre 1994

Traduire les sociolectes

URI : https://id.erudit.org/iderudit/037181ar

DOI : https://doi.org/10.7202/037181ar

Aller au sommaire du numéro

\section{Éditeur(s)}

Association canadienne de traductologie

ISSN

0835-8443 (imprimé)

1708-2188 (numérique)

Découvrir la revue

Citer cet article

Morvan, F. (1994). À propos d'une expérience de traduction : Désir sous les ormes d'Eugène O'Neill. TTR, 7(2), 63-92. https://doi.org/10.7202/037181ar

\section{Résumé de l'article}

À propos d'une expérience de traduction: Désir sous les ormes d'Eugène O'Neill. À partir d'une expérience de traduction ponctuelle (Désir sous les ormes d'Eugène O'Neill mis en scène par Matthias Langhoff en 1992), la traductrice s'efforce de rendre compte des solutions qu'elle a trouvées pour transposer l'anglo-irlandais utilisé par O'Neill comme langue de poésie. Il lui a semblé possible de traduire la dualité de cet anglais marqué par les structures du gaélique en utilisant le français marqué par les structures du breton tel qu'on l'entend encore parler en Basse Bretagne. Cependant, le recours à un parler vécu (ayant donc sa cohérence) et à une situation d'élocution semblable (de partage impossible) ont eu pour avantage, non seulement de réaliser un calque mais d'autoriser une rupture avec la norme. C'est cette rupture, acceptée en vue d'un gain d'efficacité, qui a contraint la traduction à partir des éléments ayant précisément été effacés par les autres traductions françaises de la pièce: les récurrences, le système métaphorique, la ponctuation et la graphie du texte, en tant qu'éléments constitutifs, porteurs de sens. Cet exemple très limité amène ainsi à considérer les problèmes que pose la traduction d'un sociolecte comme révélateurs d'une résistance à l'acte de traduire, inhérente à cet acte même, parce qu'issue d'une résistance à l'autre, en ce qu'il a d'étranger.
Tous droits réservés ( $\mathrm{TTR}$ : traduction, terminologie, rédaction - Les auteurs, 1994
Ce document est protégé par la loi sur le droit d'auteur. L'utilisation des services d'Érudit (y compris la reproduction) est assujettie à sa politique d'utilisation que vous pouvez consulter en ligne.

https://apropos.erudit.org/fr/usagers/politique-dutilisation/ 


\section{À propos d'une expérience de traduction: Désir sous les ormes d'Eugène $O$ 'Neill}

\section{Françoise Morvan}

S'il est toujours déplacé de parler de son propre travail, je dois tout d'abord reconnaître que, dans mon cas, c'est-à-dire dans le cas d'une expérience de traduction aussi ponctuelle et atypique que celle de Désir sous les ormes, cette tentative de réflexion m'apparaît d'autant plus périlleuse qu'elle ne s'appuie sur aucun savoir: n'étant ni spécialiste de traduction, ni spécialiste de littérature américaine, ni de théâtre, ni de linguistique, je ne peux guère qu'essayer de discerner a posteriori ce qui, sans que j'en aie eu la moindre intention, a orienté une expérience qui semble surtout intéressante dans la mesure où, échappant à tout caractère personnel, elle amène à reconsidérer le statut du texte de théâtre et témoigne d'une évolution générale des méthodes de traduction. C'est donc ce que j'essaierai de mettre en lumière à partir d'une pratique tout empirique et qui n'aura été, en somme, que le fait du hasard.

\section{Dilemmes}

\section{Les malheurs d'O'Neill}

Tout commence par une commande inopinée: ayant proposé à Mathias Langhoff de monter au Théâtre National de Bretagne une pièce en breton de Tanguy Malmanche, j'apprends soudain qu'il compte mettre en scène Désir sous les ormes d'Eugène O'Neill et qu'à trois semaines du début des répétitions il se trouve sans texte jouable. Matthias Langhoff est allemand; il a travaillé sur une bonne 
traduction allemande et, ne pouvant juger la traduction française, s'est fié au jugement des spécialistes qui lui ont dit qu'il était nécessaire de récrire le texte français, d'adapter, de rectifier. Les acteurs, confrontés à ce travail, ont déclaré forfait. On me demande de voir ce qu'il est possible de faire avec les deux versions françaises existantes, celle de Michel Arnaud, parue aux éditions de l'Arche en 1964, et celle de Ginette Herry et Claude Lacassagne, dactylographiée, plus récente mais interdite à l'édition. Le problème est précisément que les droits pour toutes les pièces d'O'Neill appartiennent aux éditions de l'Arche qui interdisent toute autre traduction que les leurs, lesquelles sont, me dit-on, injouables. La coutume est donc de jouer la traduction injouable en lui ayant fait subir un traitement approprié. Cela étant, et l'éditeur touchant les trois-quarts des droits pour un texte qui n'a parfois pas un mot de commun avec le sien, O'Neill parvient tout de même à être joué en France. On comprend qu'il le soit de moins en moins.

L'urgence aidant, je passe sur ce problème pour lire le texte et découvre cette fois une énigme: comment Matthias Langhoff a-t-il pu en venir à décider de mettre en scène ce mélodrame rural? En trois actes, l'histoire du vieux Cabot, entêté à tirer sa ferme de la terre pierreuse de la Nouvelle Angleterre, expose la révolte de ses trois fils, le départ des deux aînés pour la Californie, la lutte du plus jeune pour prendre la ferme, et son amour pour la jeune femme que le père vient d'épouser, tout cela s'achevant par un meurtre de nourrisson et un départ pour la prison sous la garde du shériff. C'est vieux, c'est long, et c'est aggravé par une sorte de patois néo-rustique quasi indéchiffrable. Prenons les premières répliques:

- Eben: God! Purty!

- Simeon: Purty!

- Peter: Ay-eh.'

Il n'est pas besoin d'aller plus avant pour comprendre qu'il y a là quelque chose d'intraduisible. Une fois admis que la langue

1. SelectedPlays of Eugene O'Neill (New York, Random House, 1988), pp. 159-160. 
d'O'Neill est l'anglo-irlandais tel qu'il a pu l'entendre autour de lui, c'est-à-dire un anglais fortement marqué par les structures du gaélique, comment traduire à la fois l'anglais et le gaélique? Comment faire entendre le gaélique en français? Ou comment restituer cette différence entre yes et ay-eh, entre pretty et purty? Il n'y a pas que la différence de prononciation, il y a tout l'écart de sens que cette différence rend sensible - purty, ça ne veut pas dire pretty - et le rythme qu'O'Neill a pris la peine de souligner dès la première de ces interminables didascalies: le texte anglais n' indique pas qu'Eben s'exclame: he blurts out with halting appreciation, écrit O'Neill, autrement dit, si la traduction est possible: il laisse échapper son admiration d'une parole hésitante - ainsi, déjà, comment traduire cette évocation du rythme d'une parole heurtée, hésitante? Aucun patois français ne convient, et si l'idée même de transposer O'Neill en normand ou en berrichon est ridicule, ça n'est pas seulement qu'un paysan berrichon n'est pas un paysan irlandais mais que, de toute façon, un patois n'est pas une langue double comme l'anglo-irlandais mais une langue autre, et à peine autre, non pas une langue duelle mais une langue à côté, légèrement à côté. Inventer une langue serait fausser les données du jeu et risquer au surplus d'être incompréhensible. Le pire serait de transposer dans ce faux langage paysan issu du picard que Molière a imposé sur la scène française comme signe même du ridicule. Alors, remettre O'Neill en bon français? Autant dire effacer tout le travail de l'auteur. Et pourquoi ce travail?

\section{Traduire O'Neill?}

Je peux dire qu'au point où j'en étais alors, ayant fermement décidé de ne pas pousser l'expérience plus avant, j'étais déjà prisonnière, et chaque question ne faisait que m'y engager un peu plus. Le choc décisif a été produit par une question sans malice, qui découlait naturellement des précédentes: cette langue intraduisible, comment le traducteur français l'avait-il traduite? La réponse était saisissante:

- Eben: Bon Dieu! C'est rien beau!

- Siméon: C'est rien beau.

- Peter: Ouais. 
On m'avait dit que c'était une traduction injouable, et je m'interrogeais sur les critères qui font qu'un texte est jouable ou ne l'est pas. En l'occurrence, jouable, la traduction l'était, sous réserve de considérer qu'écrire paysan, c'est écrire patois, qu'écrire péquenot, c'est écrire mal, et que le but d'O'Neill en transposant son bon anglais en jargon bâtard ne pouvait être que de dénonciation par le ridicule. Or, derrière ces trois répliques, qui ouvrent la pièce, ce que l'on sent, et qui rend la transposition si terrible, c'est quelque chose d'autre, quelque chose d'étrange à force d'être anodin, une sorte d'énigme. Pas de Molière, pas de Zola, pas de déformation artistique à la Jehan Rictus, pas de dénonciation sociale. Rien que cette banalité. Un cliché. Mais sans rien à voir avec ce cliché linguistique qu'impose le traducteur français pour signifier l'inaptitude à parler bien. Un cliché double lui aussi: il ne faut pas grande attention pour voir que les mêmes mots ouvrent la pièce et la ferment. Au début, Eben devant le ciel rouge du soleil couchant: God! Purty! À la fin, Eben montrant le soleil levant: Purty, hain't it? Et le même $A y$-eh pour lui répondre. En somme, face au cliché hollywoodien, un mot gauchi, maladroit, et valant pour sa maladresse: face au grandiose, à la grandiloquence, Purty pour signifier que la beauté du monde est mal dite, que la beauté des mots peut venir de ce mal dit, et prendre Dieu à témoin de ce manque.

Je suppose que c'est à ce moment-là que j'ai, non pas décidé de traduire mais entrevu la possibilité de traduire O'Neill dans la langue que l'on parlait autour de moi en Basse-Bretagne dans mon enfance - langue non reconnue, non répertoriée, ignorée de ceux-là même qui la parlent encore, et exclue de toute expression écrite, si l'on excepte toutefois quelques traductions par Tanguy Malmanche de ses propres pièces bretonnes, et notamment de ce Gurvan que je voulais faire monter par Matthias Langhoff: un français transformé par l'accentuation du breton, par sa syntaxe et par les inclusions de mots intraduisibles, sonores, brefs, qui me valaient de provoquer des rires quand je retrouvais Paris au retour des vacances. Vengeance de Malmanche ou pas, je me suis mise à tâtonner à la recherche d'un écho vague, puis d'une transposition hasardeuse, avant d'avoir pu réfléchir à ce que je faisais. Ce qu'un 
tel projet avait d'aberrant ne m'a pas frappée. Je n'ai pas même fait retour sur le théâtre de Malmanche qui aurait pourtant pu, au début tout au moins, me servir de guide, et $\mathrm{j}$ 'ai tout simplement oublié que les conditions de travail étaient inacceptables. En fait, j'avais l'impression de redécouvrir une langue connue autrefois, mais interdite, et qui, soudain permise, me rendait un monde. Si l'expérience vaut pour quelque chose, c'est peut-être pour cela.

Essayant de réfléchir aux règles qui ont pu guider un tel travail de traduction, je serais tentée d'en revenir à cette constatation initiale: si, pour moi, du premier mot de la pièce au dernier (c'est-à-dire de ces premiers mots qui, au sens strict, ouvrent et ferment la pièce) tout se joue sur l'inappropriation et ses capacités à produire du sens, et à le produire en dehors des normes, les versions françaises ne tendant pas à traduire une inappropriation mais une impuissance, une incapacité à produire normalement du sens.

Or, dès lors que nous n'avons donc pas traduit le même texte, à quoi bon comparer? Le premier traducteur français de Désir sous les ormes, Michel Arnaud, s'est voulu scrupuleusement fidèle au sens explicite du texte; on ne peut lui reprocher aucune omission, aucun contre-sens. La même honnêteté préside au travail de Ginette Herry et Claude Lacassagne. Et j'en dirais autant des traductions canadiennes qui m'ont été remises ensuite par Annie Brisset, 1'une due à Michel Dumont et Marc Grégoire, l'autre à Yves Sauvageot et Robert Ripps. En somme, tous seraient en droit de dire comme Chateaubriand à propos de sa traduction de Milton qu'ils ont calqué le texte à la vitre. Simplement, n'ayant pas lu le même texte et n'ayant pas appliqué le calque aux mêmes endroits, nous avons restitué sous le même titre des vérités incompatibles. Le propre du traducteur étant d'être aveugle à tout ce qui n'est pas sa version du texte, et donc aveugle à tout ce qui a guidé une interprétation qui se confond pour lui avec une vérité sans âge, nul autre moyen pour lui que la comparaison pour tenter de comprendre ce qu'il a fait. Qu'il soit donc entendu que la confrontation de versions différentes ne tend ici qu'à définir par différenciation les caractéristiques d'une version française parmi d'autres. 


\section{La méthode: orientations d'ensemble}

\section{Rupture avec la norme}

Reprenant ma première constatation, à savoir que les versions françaises (françaises de France) des pièces d'O'Neill donnent à entendre une langue insuffisante, c'est-à-dire volontairement dénotée par son insuffisance, je peux dire qu'en effet elle l'est par rapport à une norme qui est celle du français standard, et que, précisément, leur première caractéristique est la présence jusque dans le texte d'arrivée de cette norme à transgresser. Le maintien d'un système de référence considéré comme normal entraîne des effets, selon moi, pervers parce que tout décalage tend à produire un effet de dénonciation, soit dans l'excès soit dans le manque. Ainsi, s'agissant de l'excès, Michel Arnaud utilise-t-il le code autorisé de transgression du bon français, à savoir l'argot, pour traduire la violence: I'll kick ye both out in the road devient Je vous foutrai tous les deux à la porte et l'expression dumb fool constamment utilisée par Cabot pour désigner Eben devient un pauvre con, alors que la violence ne s'exprime jamais par l'argot dans la pièce d'O'Neill. Le manque, lui, se signale à tout instant par la présence du mal dit: qu'on se réfère simplement au c'est rien beau initial, on y verra un exemple de mal dit censé être du genre paysan; un peu plus loin, dans les paroles de Siméon sur sa femme (Moi, je $m$ 'en rappelle... de temps en temps), de Peter sur l'or (De l'or y en a à l'ouest, Sim) et des deux frères sur la terre, le rêve de partir en Californie (Des fortunes qu'est à la surface du sol et qu'attendent juste qu'on vient les ramasser) ce sont les fautes de syntaxe qui signalent le mal dit. Le problème est qu'O'Neill ne me semble pas avoir écrit dans une langue indigente, ni procédé à une dénonciation quelconque. Le texte anglais donne:

- Simeon: Purty.

- Peter: Ay-eh.

- Simeon: Eighteen year ago.

- Peter: What?

- Simeon: Jenn. My woman. She died. 
- Peter: I'd fergot.

- Simeon: I rec'lect - now an' agin. Makes it lonesome. Sh'd hair long's a hoss'tail - an' yaller like gold!

- Peter: Waal - she's gone. They's gold in the West, Sim.

- Simeon: In the sky?

- Peter: Waal - in a manner o'speaking' - that's the promise. Gold in the sky - in the west - Golden Gate - Californi-a! Golden West! - fields o' gold!

- Simeon: Fortunes layin' just atop o' the ground waitin' t' be picked! Solomon's mines, they says! ${ }^{2}$

$\mathrm{Au}$ lieu de ce texte anglais écrit en fonction de règles nouvelles - des règles à déceler d'après le contexte pour le locuteur anglais comme pour le traducteur -, on arrive en français à un deuxième type d'effets pervers qui rend le travail de l'acteur particulièrement difficile: les fautes sont d'autant plus voyantes qu'elles viennent perturber une langue dont la norme est maintenue.

- Simeon: C'est rien beau!

- Peter: Ouais.

- Simeon: Ça fait dix-huit ans.

- Peter: Quoi?

- Simeon: Jenny. Ma femme. Dix-huit ans qu'elle est morte.

- Peter: J'avais oublié.

- Simeon: Moi je m'en rappelle... De temps en temps. Et alors on se sent tout seul. Elle avait des cheveux longs comme la queue d'un cheval... des cheveux qu'étaient jaunes comme l'or!

- Peter: Eh oui... elle a passé. De l'or y en a à l'Ouest, Sim.

- Simeon: Dans le ciel?

- Peter: Ma foi... façon de causer... Dans le ciel, y en a comme qui dirait la promesse. De l'or dans le ciel... à l'Ouest... la Porte d'Or... la Californie! Y a de l'or à l'Ouest... des champs d'or!

- Simeon: Des fortunes qu'est à la surface du sol et qu'attendent juste qu'on vient les ramasser! Les Mines du roi Salomon, qu'ils disent!

2. Les didascalies ont été supprimées afin d'aller à l'essentiel, qui est ici le style adopté par O'Neill pour transcrire la langue des paysans de Nouvelle Angleterre. 
En somme, il faut tenter de rendre plausible une série d'informations contradictoires tout en faisant passer l'incohérence pour norme; une phrase aussi anodine que la phrase de Siméon, des fortunes qu'est à la surface du sol et qu'attendent juste qu'on vient les ramasser montre assez bien, parmi des centaines d'autres, l'impossibilité de concilier indigence lexicale et permanence du français écrit (ces fortunes qui sont à la surface du sol, quel paysan français les désignerait ainsi quand on dispose de l'expression à ras de terre qui est à la fois plus rapide, concrète et précise?), faute syntaxique et cohérence du système (l'absence d'accord des fortunes $q u$ 'est se voit immédiatement contredit par l'accord et qu'attendent). Si l'écart syntaxique ne peut pas être pris en compte comme donnée réelle, concrète, offrant une possibilité d'expression inédite, c'est qu'il n'est perçu que comme faute - et s'il n'est perçu que comme faute, c'est que tout opère négativement par rapport aux codes du français écrit, du bon français. La divergence essentielle est là: dans ce premier mouvement de rupture qui m'a fait, avant d'avoir écrit le premier mot, mettre hors d'usage une norme qui ne m'aurait servi à rien. Cela supposait aussi une sorte d'acte d'adhésion préalable au jargon employé par O'Neill - non seulement une acceptation, mais, justifiant le mouvement de rupture avec la langue apprise, un mouvement d'approbation entière, voire, dans mon cas, de confiance euphorique dans le choix fait par l'auteur d'une langue autre.

\section{Usage de la dualité}

En cela, je rejoins les traductions canadiennes que j'ai pu lire: ainsi l'interprétation de la première scène par Michel Dumont et Marc Grégoire donne-t-elle:

- Siméon: Jenny. Ma femme. Dix-huit ans qu'est morte.

- Peter: J'avà oublié ça.

- Siméon: Ça m'revient par escousses. J'me sens tu-seul. A avà les chfeux comme une queue de cheval, plus blonds que d'l'or! - Peter: Ouais... que c'est qu'tu veux, est partie. Siméon, parà qu'y a d'l'or dans l'ouesse.

- Siméon: Dans l'ciel?

- Peter: Ouais, le ciel, c'est la promesse de l'or, comme qu'on pourà dire. In ciel tout' en or - dans l'ouesse - Golden Gate - la 
porte de l'or - la Califournie! - L'ouesse tout' en or - des champs d'or!

- Siméon: Une fortune au ras l'sol; wa jusse à s'baisser pour la ramasser! Y appellent ça: les mines de Salomon!

Outre le vocabulaire inattendu, plaisant par l'effet de surprise et de compréhension légèrement différée (ainsi escousse dans ce passage), on retrouve O'Neill dans la cohérence de la transcription qui amène à écrire chfeux au lieu de cheveux, en accord avec une prononciation qui élide les e (il est vrai qu'on attendrait aussi chfal, alors que cheval est maintenu, mais dans le cours de la traduction chfal s'imposera) et la cohérence des terminaisons en à qui, ayant donné avà pour avait donnera parà, pourrà, c'étà.

Le fait d'écrire à partir d'un parler vécu évite au moins l'arbitraire, sert de guide contre l'incohérence et apporte, en plus de solutions en chaîne, des modèles pour ainsi dire vérifiés par l'expérience, mis à l'épreuve de la parole. On sent la langue comme un système ayant ses règles, qui sont à découvrir, et retenir par pur plaisir - or, de telles règles ne peuvent être retenues par le spectateur, ne fût-ce que pour le temps de la représentation, que s'il y croit, ce qui suppose que sa confiance ne soit pas trompée par des invraisemblances de détail comme on peut en trouver dans une reconstitution d'auteur.

Mais le risque avec l'utilisation d'un parler constitué est de s'en satisfaire: d'abord, il y a ce danger de satisfaction précisément, voire de parade ou de revendication inclus dans tout usage littéraire d'un sociolecte comme expression d'un groupe minoritaire; cet aspect n'est sans doute pas absent de l'usage de l'anglo-irlandais, mais il y a dans l'anglo-irlandais une violence qui échappe au joual, et qui vient du conflit engendré par la présence de la langue dominée dans la langue dominante, conflit interne qui à son tour engendre des formes presque constamment minées, se dénonçant comme doubles: ce qui rend si poignant l'anglo-irlandais de Synge, ce qui le rend si oppressant tel qu'O'Neill l'a systématisé, c'est cette auto-dénonciation; langue d'exil, langue de partage et de séparation, langue du partage impossible, elle contient en soi ce qu'O'Neill 
montre par ailleurs - cet impossible partage - et s'il y a quelque chose de véritablement puissant dans la pièce, c'est cette inscription de la mise en scène dans la langue. En somme, traduire un sociolecte, c'est d'abord traduire une situation d'élocution. Dans les traductions canadiennes, je lis (à tort ou à raison) une sorte de certitude et d'affirmation pleine radicalement étrangère aux textes d'O'Neill. Et je dirai même que ces mots si riches, si séduisants, tendent parfois à un pittoresque qui n'est présent dans O'Neill que pour être dénoncé, pulvérisé de l'intérieur par cette négation féroce qui rend aussi l'intrigue possible. En changeant cet état d'exil lisible dans le nom même de Cabot ${ }^{3}$, on attaque la trame d'ensemble de manière certainement invisible mais d'autant plus agissante. À de tels détails on peut voir aussi que traduire un sociolecte n'est jamais seulement traduire une langue mais un texte qui la met en scène, inséparablement de la représentation dans son tout - or, plus la pièce a de valeur littéraire, plus est grande la cohérence des éléments qui la composent: les personnages d'O'Neill sont une émanation de leur syntaxe, et il n'est pas interdit de penser que l'intrigue est une mise en scène de la langue inventée par O'Neill.

\section{La dualité pour retour au texte}

Une langue inventée - on touche par là à ce qui, dans les traductions que je pourrais dire «véristes», si drôles, si touchantes, si parlantes soient-elles, risque de les empêcher d'être pleinement convaincantes:

3. Le nom du vieux Cabot est une allusion à Jean Cabot qui, ayant débarqué en Amérique en 1497, explora les côtes du Labrador et de la Nouvelle Angleterre, faisant ainsi figure de père fondateur, de premier père né de l'exil. À cette allusion à l'exil répondent évidemment par antithèse - le symbole se dissimulant derrière la coutume très répandue de donner des noms bibliques - les noms de Peter et de Simeon (Tu es Pierre et sur cette pierre je bâtirai mon église, dit le Christ à Simon Pierre dans l'Évangile selon Saint Mathieu) équivalant à celui d'Eben (Ebenezer: pierre) comme négation de l'exil par son inscription dans la pierre. Il est difficile de ne pas voir que l'itinéraire d'Eben, de la pierre à l'exil, reprend celui du père en sens inverse, orientant ainsi tout le sens de la pièce. 
un dialecte bien maîtrisé par le traducteur tend naturellement à s'imposer, avec ses règles, ses structures propres, et à substituer au système d'origine un système, en cela aussi, auto-suffisant, c'està-dire assez satisfaisant pour faire pléthore. Le problème est d'abord qu'O'Neill n'a pas copié un sociolecte; il l'a réinventé, travaillé, retravaillé, de manière à le rendre audible aux spectateurs. Lorsque je lis le monologue du vieux Cabot sur les pierres, ou tout fragment de récitatif de ce genre, je sais que ce qui importe là, c'est le rythme, et je suis obligée de savoir aussi que l'anglo-irlandais a été employé par O'Neill, non pas sans doute uniquement parce qu'il lui offrait la possibilité de donner vigueur à ce rythme mais de manière à lui permettre de le faire entendre, en sorte que la langue est constamment gauchie. Si je traduis en me guidant sur les structures du dialecte qui est le mien, et si, au lieu d'accepter de les perdre, je les impose, à ce moment-là je perds l'essentiel. Le texte anglais donne:

Listen, Abbie. When I come here fifty-odd year ago - I was jest twenty an' the strongest an' hardest ye ever seen - ten times as strong and fifty times as hard as Eben. Waal - this place was nothin' but fields o'stones. Folks laughed when I tuk it. They couldn't know what I knowed. When ye kin make corn sprout out o' stones, God's livin'in yew. They wa'n't strong enuf for that! They reckoned God was easy. They laughed. They dont' laugh no more.

Il est inutile de s'attarder sur la traduction autorisée:

Écoute, Abbie. Quand je suis arrivé ici, y a quelque chose comme cinquante ans... J'avais juste vingt ans et j'é tais le gars le plus costaud et le plus dur que t'aies jamais vu... dix fois plus costaud et cinquante fois plus dur qu'Eben... Eh ben... Cette ferme, c'était rien que des champs de pierre.

Sans même tenir compte de l'incongruité de l'écho Eben/Eh ben, le texte se donne pour de la prose, une mauvaise prose, lourde, lente, qui n'a rien à voir avec la scansion biblique de l'anglais. 
lent:

Le texte de Michel Dumont et Marc Grégoire est également

Écoute-moé, Abbie. Quand j'm'en sus t'en'nu de par icitte, y a cinquante ans et quèque de d'ça, j'avà jusse vingt ans pis j'étà fort pis dur comme y s'en fâ pus - dix fois - plus fort que Eben pis cent fois dur comme lui.

La version d'Yves Sauvageot et Robert Ripps est beaucoup plus efficace mais il lui manque le principe organisateur qui est le rythme:

Abbie, écoute. Quand ch'arrivé ici ya à peu près d'ça, cinquante ans... j'avais juste vingt ans, pis le plus fort pis l'plus solide que t'as jamais vu... dix fois plus fort pis cinquante fois plus solide qu'Eben. C'te lot était rien d'autre qu'un champ de roches...

Dans les deux cas, le recours à des structures préexistantes est une entrave, qui empêche de comprendre que le texte est construit à partir d'une scansion matérialisée dans le texte par une ponctuation obsédante - cette ponctuation que tous les traducteurs ont commencé par effacer et qui a été, pour moi, le point de départ de mon travail, c'est-à-dire d'un travail qui n'était pas de reconstitution d'un sociolecte mais de travail du souffle grâce à la liberté que m' offraient les structures ouvertes par l'usage d'un parler non fixé:

Listen, Abbie./

When I come here / fifty-odd year ago -

I was jest twenty /

Quand j'entends ce début, je sais que j'ai d'abord à transcrire cet exorde: $2 / 2 / / 4 / 6 /$ et qu'il me faut trouver un rythme qui donne cet élan, cet allongement progressif, et qui amène à reconnaître au fil des paroles du vieil Ephraïm le retour de la scansion majestueuse de la Bible. Je me souviens parfaitement du moment où, ayant jusqu'alors traduit en appliquant plus ou moins mécaniquement les structures du breton, j'ai commencé à sentir ce rythme et à écrire en alexandrins, c'est-à-dire à écrire avec pour 
base un alexandrin rompu qui me permettait, à partir de cette base maintenue, soit d'allonger les séquences successives, comme O'Neill le fait très souvent, soit de les casser:

Écoute / Abbie. / $2 / 2$ /

Quand ça j'suis v'nu ici / c'est vieux d'plus d'cinquante ans - 6 16

vingt ans j'avais / 4

et fort $/ 2$

et dur $/ 2$

comme t'en as pas vu deux / 6

À ce moment-là, ce qui m'a servi n'a plus du tout été la possibilité de produire du décalage, plus ou moins arbitraire, grâce à un parler pittoresque, mais d'user du breton pour sa capacité à gagner des syllabes, à gagner par là en efficacité, pour l'économie véritable qu'il autorisait: vingt ans j'avais, c'est du breton traduit, mais cela n'a rigoureusement aucune importance, cela pourrait aussi bien être du hottentot sans que cela change rien à l'essentiel qui est ici la rapidité, le gain dû à l'ellipse et à l'inversion.

Jusqu'alors, j'avais cru être fidèle à O'Neill en traduisant une langue duelle, une langue paysanne, ce en quoi je voyais les deux caractéristiques majeures de l'anglo-irlandais d'O'Neill. Il y manquait la caractéristique première, à savoir qu'il s'agissait d'une langue de poésie, que cette poésie naissait d'abord d'un rythme qui obligeait à repenser la phrase tout entière et qu'il suffisait d'une syllabe de trop ou de moins pour que tout soit manqué. Je pense que si le breton m'a été utile à ce moment-là, ç'a été parce qu'il ne s'imposait pas. D'une part, il s'agit d'une langue à syntaxe très souple, qui a pour règle de placer en tête de phrase l'information sur laquelle on souhaite insister, ce qui offre une grande liberté; d'autre part, loin de me sembler établie, porteuse de vérité stable, c'est une langue que j'utilisais là pour la première fois, et de manière tout à fait hasardeuse, sans trop y croire. Elle m'a offert cette possibilité, précieuse entre toutes, de la quitter pour me rapprocher de la langue d'origine: j'ai gardé trace des tâtonnements successifs à la recherche d'une version acceptable; tous tendent à fabriquer, à partir du français, mais d'un français reconstruit de l'intérieur par le breton, 
une langue qui adopte la scansion du texte anglo-irlandais et qui transmette quelque chose de son énergie.

J'en arriverai ici à une troisième conclusion de l'expérience, qui est que traduire un sociolecte au théâtre, ça n'est pas seulement traduire une situation d'élocution, et traduire un texte qui la mette en scène, mais surtout traduire ce qui a fait qu'il a été choisi par l'auteur de préférence à toute autre langue, c'est-à-dire traduire ses particularités en tablant sur le fait qu'elles sont un gain et qu'il faut d'abord faire en sorte de gagner en efficacité grâce aux équivalents à trouver; autrement dit, porter dans le travail de traduction la même confiance aveugle qui a fait d'entrée de jeu admettre que l'auteur avait dû choisir de rompre avec la norme pour des raisons nécessairement primordiales.

\section{La méthode: approche du texte}

Ce que ma méthode de traduction a pu avoir de particulier se résume donc en cette confiance aveugle: acceptant d'entrée de jeu les moindres bizarreries de l'auteur, je les ai transposées, en attendant de comprendre. Cela supposait, en plus de la confiance, un bon logiciel de traitement de texte et beaucoup de patience. Il est arrivé que la raison d'être de telle ou telle répétition ne se révèle que tout à la fin de la pièce et qu'il faille alors réévaluer toutes les occurrences, ce qui voulait dire récrire la plupart des phrases où elles apparaissaient et qui se trouvaient déséquilibrées par le changement. Cela suppose aussi que les défauts du texte soient pris en compte, non comme défaillances mais comme éléments constitutifs, ce qui s'oppose, bien sûr, de manière radicale à la tradition française.

\section{Récurrences}

La langue d'O'Neill est pauvre; celle de Racine aussi, mais elle est noble; jugera-t-on que celle d'O'Neill est également noble, voire hiératique, cela n'empêchera pas qu'on y trouve ce corollaire de la pauvreté qu'est la répétition: preuve d'indigence, blâmable en soi et, qui plus est, portée par O'Neill à des degrés insupportables. Rien 
d'étonnant que tous les traducteurs effacent de leur mieux ces répétitions de même qu'ils rétablissent l'orthographe et rectifient la ponctuation. Il m'a semblé que la répétition valait précisément pour son côté obsédant, insupportable: il n'y a pas que ce purty qui ouvre et ferme la pièce mais tous ces mots porteurs du sens profond de la fable, qui, par la répétition, prennent valeur d'emblème, et qui apparaissent, par leur sonorité même, comme des doublets - god, gold, sun, sin, stone, steal/stole. Non seulement effacer la récurrence serait étouffer ces émergences d'une signification à la fois mise en évidence et cachée dans les mots, mais modifier la place qui leur était assignée reviendrait à effacer des rencontres, des confrontations, qui sont également constitutives du sens et de ses variations.

Passé un certain moment, il est devenu évident que la répétition ne touchait pas seulement les mots, même si le retour de certains mots était ce qu'il y avait peut-être de plus ostensible dans la pièce, mais des fragments entiers, puis des phrases. Ainsi les paroles du vieux Cabot, reprises par ses fils, étaient-elles des réserves de leitmotive:

C'est le printemps, qu'y dit, et $\mathrm{j}$ 'suis comme un damné. Comme un vieux noyer noir damné d'à êt' brûlé, qu'y dit. Et là probab'qu'y voit qu'j'ai comme un air d'espoir, du coup le vlà tout r'quinqué mauvais: «Va pas penser qu'j'suis mort», y fait, «j'ai juré d'aller diqu'à cent, sûr j'irai, seraye-c'que pour tuer vot' coupab' convoitise»! Et maint'nant j'm'en vais pour connaît' le message que Djieu y m'a mis dans l'printemps, ainsi comme les prophètes.

On peut isoler des thèmes: le thème comme un vieux noyer noir, ou encore j'ai juré d'aller diqu'à cent, sûr j'irai qui répond à son opposé $j$ 'me fais vieux, $j$ 'suis l'fruit du bout d'la branche et la coupab' convoitise qui devient la dénonciation de l'or coupab' de même que le thème biblique et maint'nant j'm'en vais pour connaît' le message que Djieu y m'a mis dans l'printemps annonce toute une série de formules dérivées de la Bible qui se rassemblent dans le grand monologue de Cabot: 
I could o'been a rich man - but somethin' in me fit me an' fit me - the voice o' god sayin': "This hain't wuth nothin' t' Me. Git ye back t'hum!» I got afeerd o' that voice an' I lit out back t'hum here [...] God's hard, not easy! God's in the stones! Build my church on a rock - out o' stones an' I'll be in them! That's what he meant t'Peter! Stones. I picked 'em up an' piled 'em into walls. Ye kin read the years o' my life in them walls, every day a hefted stone, climbin' over the hills up ans down, fencin' in the fields that was mine, whar I'd made thin's grow out o' nothin' like the will o' god, like the servant o' His hand. It wa'n't easy. It was hard an' he made me hard fut it.

...mais dans l'dedans, ça m'rev'nait, ça m'cognait - la voix d'Djieu qui m' disait «Tout ça n'veaw rien pour Moi. R'tourne à la maison!» J'ai eu peur d' cette voix, et j'ai tout laissé pour m'en r'tourner à la maison ici [...] Djieu, l'est dur, l'est pas facile! Djieu l'est dans les pierres. Et sur cette pierre j' bâtirai Mon église - toute avec des pierres et j's'rai dans ces pierres. C'est c'qu'il a dit à Pierre. Les pierres. J'les ai tirées, j'les ai mises dans des murs. Tu peux lire les années d'ma vie dans ces murs, chaque jour une pierre de plus, à r'monter des collines ou r'descendre, à clore les champs qu'étaient à moi, où j'avais tout fait pousser d'rien comme la volonté d'Djieu, comme l' serviteur d' Sa main. C'était pas facile. C'était dur et Djieu, y m'a fait dur pour ça.

Pour savoir précisément comment traduire ces récurrences, en fonction de contextes qui peuvent varier, il a fallu attendre, avancer, parfois jusqu'à la fin du dernier acte, quitte à tout reprendre à rebours, en isolant les occurrences et en procédant à des arbitrages en fonction de l'ensemble. Peu à peu, certaines solutions, adoptées provisoirement, se sont avérées plausibles; d'autres, une fois revues, ont trouvé place dans ce qui devenait un véritable puzzle, le texte du dernier acte se travaillant sur ordinateur en mode copier/coller comme un véritable collage de citations: les dernières répliques, et particulièrement celles du vieux Cabot, sont en fait un montage.

Djieu l'est dur, l'est pas facile! Pt' êt' bien y a d'l'or facile dans l'Ouest, mais c'est pas d'l'or de Djieu. L'est pas pour moi. J'entends Sa voix qui m' met en garde encore d'êt' dur et d'rester dans ma ferme. J'vois Sa main qui s'sert d'Eben pour m'voler et 
m'garder d'êt' faib'. Et j'me sens pris dans la paume de Sa main, et Ses doigts y m'guident. [...] Ça s'ra bien plus seul maint'nant qu'ça été tout d'avant - et j'me fais vieux, Seigneur - d'au bout d'la branche... [...] Bôn - et qu'est-c'tu veux? Djieu l'est seul, pas vraye? Djieu, l'est dur et seul!

Ce que le texte a de force tragique, il le doit à la répétition de fragments qui deviennent là ce qu'ils sont - des citations auxquelles on revient et qui vous enferment, pierre sur pierre, comme les murs; réduit à se répéter lui-même, le vieux Cabot n'est plus que cette immobilité de pierre qui le ramène à un dieu vide, réduit à une affirmation grandiloquente parmi d'autres - et c'est pourtant là, en cela, que se manifestent une douleur et une grandeur réelles, d'autant plus réelles qu'elles ne s'appuient sur rien et que la ferme, désertée par tous, ne lui laisse que son vide. Si la méthode qui consiste à traduire un mot par un équivalent constant est assurément contestable dans la plupart des cas, il m'a semblé qu'un tel texte rendait pourtant ce choix obligatoire: loin d'être preuve de déficience, la répétition amenait le texte à creuser sa propre antériorité, à s'inscrire dans une histoire, et à le faire précisément sur le mode de la répétition, d'une répétition de plus en plus obsédante, matérialisant dans le texte cet enfermement des personnages dans une parole qui ne se renouvelle que pour se redire, matérialisant dans la langue la métaphore qui porte la pièce tout entière.

\section{Métaphores}

C'est en ce point ultime où se rejoignent la répétition et la trame métaphorique qui sous-tend le texte que leur nécessité apparaît le mieux: impossible d'anéantir une récurrence sans porter atteinte à un réseau dont tous les éléments sont solidaires. La présence de Dieu, elle apparaît dans cette exclamation qui est le premier mot de la pièce mais aussi dans les exclamations qui la ponctuent, Tonnerre de Dieu! et toutes ses variations qui ne sont pas non plus dénuées de signification, Dieu Tout Puissant! n'ayant pas le même usage que Bon Dieu! Autant de jurons, autant de blasphèmes, qui, à leur tour, ne se comprennent que par rapport aux allusions au diable: God 
A'mighty, I got enuf. Ye got the devil's strength in ye, dit le violonneux à visage maléfique au moment où le vieux Cabot l'emporte dans l'épreuve, véritablement diabolique (on trouve là un écho des contes irlandais) d'accélération de la danse. Et Eben, chassant Abbie: T'es qu'une putain - une sal 'té d'putain d'ruse du djiab'! Les allusions au diable sont redoublées par les injonctions constantes à filer en enfer - à s'en aller au diable. Où c'est qu'il a djiab' pu aller, tu penses, Sim? demande Peter dès le début: quitter la ferme, c'est forcément aller au diable, l'or de l'ouest, signifiant toujours tentation, perdition, comme la Minnie du bout de la route, la Femme Écarlate, et tout ce qui s'oppose à la pétrification, à l'enfermement dans la stérilité divine de la pierre.

Cette géographie imaginaire de la pièce lui est aussi consubstantielle que la manière dont les personnages se définissent eux-mêmes ou définissent les autres par des images-totem: comme un vieux noyer noir brûlé d'à être damné, dit Cabot de lui-même; et ses fils, le vieux putois, le vieux serpent, la vieille mule; alors qu'Eben est pour Cabot un pauv' gobe-lune, doux comme chiffe. Le jeu serré qu'entretiennent ces images apparaît à certains moments de rencontre - ainsi quand l'horreur qu'inspire la nouvelle femme du père s'exprime pour Eben comme une horreur du père, par le biais des images-totem qui le définissent: $\mathrm{Her}$ - here - sleepin' with him - stealin' my Maw's farm! I'd as soon pet a skunk 'r kiss a snake! (Celle-là-ici - à coucher avec lui - à voler la ferme à ma Mère! Autant moumouner un putois, embrasser un serpent!) Et l'on voit que, pour Eben, s'emparer de la «nouvelle mère», c'est d'abord s'emparer du père.

Le mot moumouner offre l'un des rares exemples où le breton donnait un équivalent transposable directement (le verbe moumounañ signifie précisément to pet qui n'a pas d'équivalent français) et accessible à la compréhension; plusieurs tournures d'emprunt ont permis ainsi un gain d'efficacité mais, en définitive, si le breton m'a été utile, ça n'a pas été pour le vocabulaire, ni même pour la syntaxe à proprement parler, mais pour la possibilité, ayant une langue non fixée, d'utiliser cette ouverture pour éliminer toute notion de faute - la faute devenant moyen d'inventorier des 
possibilités non exploitées de la langue - et de respecter ce qui était l'essentiel à mes yeux, c'est-à-dire la ponctuation comme principe organisateur.

\section{Ponctuation}

Ayant commencé à traduire Désir sous les ormes à partir du moment où, entendant soudain le rythme du monologue de Cabot sur les pierres, j'ai senti qu'il me fallait inventer une langue, propre à servir ce rythme, et non pas une syntaxe, il me faut m'arrêter un peu sur la première particularité de cette traduction puisqu'elle constitue aussi, comme on l'a vu, le premier point de divergence avec les autres traductions. Il me semble bien pouvoir dire toutes les autres traductions, car, si Ginette Herry et Claude Lacassagne restituent les tirets qui ponctuent le texte, ils semblent les placer sur le texte comme éléments décoratifs plus que comme éléments véritablement constructifs:

- Peter: Here - it's stones atop o'the ground - stones atop o' stones - makin' stone walls - year atop o' year - him 'n' yew 'n' me 'n' then Eben - makin' stone walls for him to fence us in!

C'est en fait à partir de la version de Ginette Herry et Claude Lacassagne que l'on entrevoit le mieux le rôle de la ponctuation dans le texte d'O'Neill:

- Pierre: Ici, des pierres, par terre - pierre après pierre - faire des murs de pierre - année après année - lui et toi et moi et puis Eben - faire des murs de pierre pour que lui nous enferme dedans.

La traduction a beau être fidèle au sens et les tirets placés où $O$ 'Neill les place, l'impression de longueur, de flou, et de fluidité amène à voir que les tirets ne sont pas là seulement comme des pauses marquant la scansion d'une parole heurtée (on se souvient de la première didascalie: Eben exprime son admiration d'une parole qu'O'Neill qualifie de halting mais comme des pauses servant à noter les points où la pensée bute, contre le refus de dire, et la crainte du silence. Si je traduis $I c i-c$ 'est d' la pierre qu'y a-et 
pas à ras d' terre - d'la pierre su' d' la pierre - d'quoi faire des murs de pierre - et des années sur des années de murs de pierrelui, toi, et moi, avec Eben en plus - à faire des murs de pierre pour qu'y nous ferme dedans! c'est pour traduire le heurt, la progression de la pensée par raccrocs, et l'effet coup de poing final, avec ce décasyllabe en anglais qui ramène en français à l'alexandrin comme au mètre obligé de la tragédie.

Cela vaut pour l'ensemble du texte mais il y a aussi un usage beaucoup plus complexe de la rupture, rendant le rôle de la ponctuation également plus difficile à transposer et obligeant à travailler toute la phrase à partir du heurt instauré: If that's what his coming 's done t' me - killin' yewr love - dit Abbie à Eben, parlant de son fils. Killing yewr love - l'essentiel est ce retrait brutal, et cette rupture qui contient déjà, non dite, la possibilité de tuer le bébé. Si c'est tout c'qu'il m'a fait en v'nant-tuer ton amour - pour un bretonnant de naissance, la structure de la phrase est normale; peu importe d'ailleurs qu'elle le soit ou non, l'essentiel est l'ellipse qui signifie en soi, en dehors de toute autre information, l'importance de ce qui est dit. Cela, la syntaxe du français ne le permet presque jamais: elle retarde l'expression, elle allonge ce qui ne vaut que pour être dit en quatre syllabes ( $S i$ c'est ça que sa naissance, elle a fait pour moi... si elle a tué ton amour... écrit Michel Arnaud). Et surtout elle amène à établir un parallèle qui est radicalement opposé à la syntaxe d'O'Neill (Si c'est ça l'résultat pour moi - s'il tue ton amour - écrivent Claude Lacassagne et Ginette Herry). Or, dès lors que l'on prend en compte ces scansions et ces ruptures, il devient évident que la matière même du texte est à travailler comme un tout sonore.

\section{Graphie}

Peut-on écrire: Ici - c'est de la pierre qu'il y a-et pas à ras de terre - de la pierre sur de la pierre - de quoi faire des murs de pierre - sans écrire faux, c'est-à-dire sans fausser le rythme? A la nécessité de guider le travail de l'acteur s'ajoute la nécessité d'être cohérent: il y a est impossible, il faut donc penser le texte en fonction d'une économie apportée par la suppression du $i l$, qui, en 
effet, ne sert pas; sitôt la première élision marquée (conformément d'ailleurs au modèle proposé par O'Neill), on se trouve en présence d'un texte qui se signale graphiquement comme étant hors norme: Ici-c'est d' la pierre qu'y a-et pas à ras d' terre ce n'est pas Ici, c'est de la pierre qu'il y a, et pas à ras de terre. Mais Ici-c'est d' la pierre qu'y a, ce n'est pas autre chose qu'un texte mis comme entre guillemets, aussi ostensible, aussi peu satisfaisant que les retranscriptions de parlers paysans que l'on trouve dans les romans de la comtesse de Ségur, le parler rural se signalant par un fourmillement d'apostrophes, un texte criblé d'allusions à des manques. La seule manière d'échapper au pittoresque ne serait-elle pas - comme pour la syntaxe - de basculer, de changer complètement la façon de voir le texte? Élider les e, non pour marquer la bizarrerie mais pour montrer où poser la voix, amène aussi à transposer une prononciation, non pour faire pittoresque, mais pour signaler un système phonologique comme on transcrit une musique différente dans sa totalité. Le fait qu'O'Neill ait éprouvé le besoin de retravailler son texte en fonction de cette musique différente, et que ce texte doive sa beauté d'avoir été travaillé comme une partition, conduit naturellement, et pour que ce texte soit autre chose qu'une parodie, à tenter une reconstitution en fonction des notations incluses dans l'original. Pour moi, je disposais de quelques indices - la diphtongaison de yewr, par exemple, la palatalisation de Indjians - qui me rappelaient des traits phonologiques du dialecte vannetais que l'on parle au sud de mon village; dialecte qui offrait une solution avantageuse pour plusieurs raisons: d'une part, au contraire de tous les autres dialectes bretons, il est accentué sur la dernière syllabe; d'autre part, musical, doux, avec une sonorité nostalgique, un peu discrète, qu'Armand Robin qualifiait de féminine dans sa présentation de sa traduction du poète vannetais Yann-Ber Kalloc' $h$, il me semblait correspondre à la présence de la langue irlandaise dans l'anglais comme d'une féminité perdue, qui est aussi une expression profonde de la mère perdue, de la mère patrie, de cette mère qui revient comme un fantôme dans la pièce vide. J'étais arrivée à une version vannetaise - qui me semble, maintenant encore, plus satisfaisante que la version définitive, car la palatalisation en dj était justifiée par celle de certains $\mathbf{k}$ en tch et la diphtongaison en aw appuyée par des 
terminaisons en eye: D'vreyesfortunes tch' y a là... - mais, une fois parvenu à ce point, il aurait fallu à nouveau poursuivre, et envisager le travail du texte en fonction de cette trame à déchiffrer par la voix.

Placés devant cette trame indéchiffrable, les acteurs avaient d'abord un mouvement de recul - Qu'on me le traduise, a demandé Jean-Marc Stehlé, l'acteur chargé de jouer le rôle du vieux Cabot. Mais, traduire, c'était justement dire le texte, le faire passer par une voix qui l'interprète en cherchant comment changer aussi les points d'articulation, la manière d'accentuer, en inventant une diction comme O'Neill avait inventé un parler écrit. J'ai pu là vérifier que donner à un acteur un texte jouable, ça n'est pas lui donner un texte facile mais, s'il le faut, un texte qui résiste, c'est-à-dire qui résiste en lui offrant prise, qui ne le place pas en face d'informations contradictoires (le passage du registre écrit au registre argotique dans une même réplique, par exemple). S'appropriant la musique de la phrase, l'acteur le plus rétif finissait par trouver plaisir à dire un texte immuable comme un vers de tragédie classique, tout en cherchant sa manière propre de lui donner vie. À partir de là devenait possible tout un travail de la voix, du souffle, de l'articulation et de l'accentuation qui aurait pu faire partie du spectacle au même titre que le travail sur la langue du texte - et j'observe que la transcription vannetaise, si excessive qu'elle ait pu paraître, était plutôt en retrait par rapport au texte d'O'Neill, beaucoup plus éloigné de l'anglais courant que toutes les versions françaises; seulement, et c'est aussi une des leçons de l'expérience, de même qu'il était impensable de demander aux acteurs de travailler la matière sonore de la langue pour arriver à une unité de prononciation, fictive ou non, inspirée ou non du vannetais, il était impossible de garder le texte dans son état final, et la version qu'on lira ci-après est donc une version moyenne, standardisée à son tour, et qui, bien qu'interdite à l'édition, s'est efforcée d'avoir, en quelque sorte, l'air présentable. Cette impossibilité me semble intéressante dans la mesure où elle est le corollaire de la demande qui a provoqué cette expérience de traduction.

Le fait qu'un tel travail se soit effectué à partir de points de basculement aussi précis et aussi apparemment ténus que la 
ponctuation, et que la moindre évolution du texte ait produit des effets de modification massifs obligeant à repenser toute la traduction dans son ensemble, incite à réfléchir aux conditions dans lequelles il a été effectué: était-il possible de traduire O'Neill en faisant ce qu'on me demandait, à savoir procéder à une synthèse des versions existantes pour les rendre jouables? Peut-être le fait d'être jouable recouvre-t-il beaucoup de critères mais peut-être aussi n'exprime-t-il qu'une nécessité pratique de faire passer un texte, en le rendant, justement, passable, c'est-à-dire normal, ce qui, dans le cas d'O'Neill, revenait à l'annuler. En juger serait trancher un problème qui, pour moi, reste posé dans toute son acuité: traduire, est-ce effectuer un travail d'interprétation supposant une mise en œuvre de qualités qui ne sont guère différentes de celles de l'auteur, ou pratiquer une activité clandestine, parasite, à laquelle on ne demande rien d'autre que de rester inaperçue? Il me semble que, sauf à dérouter l'attente, comme je me suis trouvée amenée à le faire, sans d'ailleurs l'avoir du tout voulu - simplement parce qu'étant étrangère à la profession, je n'avais d'autres présupposés que le texte qui m'avait été soumis - le traducteur littéraire se trouve répondre à une demande trop contradictoire pour autoriser la pratique d'un métier véritable. Le fait qu'un auteur comme O'Neill puisse être, jusqu'à son entrée dans le domaine public, soumis à une traduction unique, sans doute inadéquate, mais dont la qualité n'a aucune importance, dût-elle ridiculiser l'auteur qu'elle est censée servir, en est la meilleure preuve; et j'ajouterai pour finir que cette preuve se voit à son tour confirmée à lire le dossier de presse: sur près de cinquante critiques de théâtre amenés à rendre compte de la représentation, quatre semblent avoir vu que le texte français joué n'était pas celui des éditions de l'Arche, aucun ne cite ce texte sans le tronquer et le seul critique qui s'attarde un peu à présenter une traduction nouvelle qui le transporte d'enthousiasme évoque ensuite avec le même enthousiasme les amours de personnages qu'il baptise Ebel et Abbie Putman. En conclusion, l'inaptitude à traduire les sociolectes n'est que la manifestation la plus visible, la plus aiguë, d'une résistance à l'acte de traduire - et peut-être le problème est-il d'abord passionnant par le champ de recherches qu'il ouvre. 


\section{Eugène O'Neill \\ Désir sous les ormes \\ Traduction de Françoise Morvan ${ }^{4}$}

Nota: Cet exemple très limité semble intéressant dans la mesure où il amène à considérer les problèmes que pose la traduction d'un sociolecte comme révélateurs d'une résistance à l'acte de traduire.

\section{Scène 1}

L'extérieur de la ferme. Le soleil couchant, au début de l'été 1850. Tout est calme; il n'y a pas de vent. Le ciel, par dessus le toit, est éclairé de couleurs intenses, le vert des ormes s'embrase, mais la maison, dans l'ombre, semble par contraste pâle et déteinte. Une porte $s$ 'ouvre; Eben Cabot s'avance jusqu'au bout de la véranda et se tient là, regardant à droite, vers la route. Il porte une grosse cloche qu'il agite d'un geste machinal, provoquant ainsi un vacarme assourdissant. Après quoi, les mains sur les hanches, il reste contempler le ciel. Troublé, saisi d'une crainte mysté rieuse, il soupire et, brusquement, traduit son admiration d'une parole hésitante.

- Eben: Bon Djieu! C'est beaw!

ll baisse les yeux et, le front plissé, scrute les environs. Âgé de vingt-cinq ans, il est grand et musclé, avec un visage avenant aux traits réguliers, assombri par une expression méfiante et rancunière. Ses yeux sombres, pleins de défi, font penser à ceux d'une bête sauvage en captivité. Chaque jour est pour lui une cage dont il resterait prisonnier, mais insoumis intérieurement. On sent en lui une vitalité farouche, toute réprimée. Il a des cheveux noirs, une moustache, un léger collier de barbe bouclée. Il porte les grossiers vêtements de travail des fermiers.

Après avoir craché par terre d'un air profondément dégoûté, il fait demi-tour et rentre dans la maison.

Siméon et Peter s'en reviennent après leur journée de travail à la ferme. Ce sont des hommes de haute taille, beaucoup plus agés que leur demi-frère (Siméon a trente-neuf ans, Peter trente-sept) et plus

4. Traduction inédite. 
carrés que lui, plus frustes, plus lourds de corps, de visage plus ordinaire et plus bovin, d'expression plus rusée, plus terre à terre. Ils avancent d'un même pas pesant, un peu voûtés par les années de travail à la ferme, traînant leurs gros souliers aux épaisses semelles incrustées de terre. Leurs vêtements, leurs visages, leurs mains, leurs cous et leurs bras nus sont souillés de terre. Ils sont imprégnés de l'odeur de la terre. Arrêtés ensemble devant la maison, comme obéissant à la même force, ils restent, silencieux, appuyés sur leurs houes, le visage levé, à contempler le ciel. Leurs visages ont une expression fermée, rétive, qui s'adoucit au fur et à mesure.

- Siméon (à contreccur): C'est beaw.

- Peter: E-waye.

- Siméon (brusquement): Dix-huit ans ça fait.

- Peter: Quoi?

- Siméon: Jenn. Ma femme. Qu'elle est morte.

- Peter: J'avais pus souv'nir.

- Siméon: Ça m'revient - des fois. M'fait tout vide. Des ch'veux longs tant qu'la queue d'un ch'val - et blonds tant comme l'or!

- Peter: Bôn - elle est pus. (Cela, sur le ton indifférent et péremptoire de l'évidence-puis, après une pause) Y a d'l'or dans l'Ouest, Sim.

- Siméon (encore sous l'impression du coucher de soleil -d'un air vague): Dans l' ciel?

- Peter: Bôn - façon d' dire - là qu' s'raye la promesse. (s'animant) De l'or dans l'ciel - dans l'Ouest - la Porte d'Or - la Cal'fornie! - l'ouest rempli d'or! - les champs d'or!

- Siméon (s'enflammant à son tour): D' vrayes fortunes qu'y a là, juste à ras d' terre, qu'à se baisser pour prend'! Les mines au roi Salomon, à c'qu'y disent. (Ils continuent un certain temps à regarder le ciel - puis baissent les yeux).

- Peter (amer et sardonique): Ici - c'est d' la pierre qu'y a et pas à ras d' terre - d'la pierre su' d' la pierre - d'quoi faire des murs de pierre - et des années sur des années de murs de pierre - lui, toi, et moi, avec Eben en plus - à faire des murs de pierre pour qu'y nous ferme dedans!

- Siméon: Tant d'travail qu'on a fait. Nos forces qu'on a données. Nos années. En labours dans la terre - (il frappe la 
terre du pied, d'un geste de révolte) - à pourrir dedans - faire $\mathrm{du}$ fumier pour grossir ses récoltes! (Une pause) Bôn - la ferme donne bien pour l' pays.

- Peter: D' ces labours-là, en Cal'fornie, c'est d' l'or en barre qu'en viendraye!

- Siméon: La Cal'fornie, c'est l'aut' côté du monde, 'tant dire. $\mathrm{Y}$ feaw bien calculer -

- Peter (après une pause): J' trouveraye dur aussi d' perd' tout ça qu'on a gagné ici à la sueur d' not' front. (Une pause. Eben passe la tête par la fenêtre de la cuisine et reste écouter.)

- Siméon: E-waye. (Une pause) Pt-êt' bien - y s'ra mort bientôt.

- Peter (d'un air sceptique): Pt-êt' bien.

- Siméon: Pt-êt' bien - va savoir - l'est mort déjà.

- Peter: Faudraye la preuve -

- Siméon: Deux mois qu'il est parti - et pas un mot d'lui.

- Peter: Nous a laissés aux champs, un soir tout comme çui-ci. Att'lé la jument, parti droit dans l'ouest. C' pas chrétien c't'histoire. Trente ans ou plus qu'il avait pas mis le pied en d'hors d' cet' ferme, d'puis l'temps qu'l' avait marié la mère d'Eben. (Une pause. D'un air rusé) J'calcule qu'y auraye moyen d'faire dire au juge qu'il a tourné fou.

- Siméon: Les a tous trop bien écorchés. Plumés diqu'à l'os. Aucun ira croire qu'il a pu tourner fou. Feaw attend'- qu'on l'aura mis en terre.

- Eben (avec un petit rire sarcastique): Ton Père honoreras! (Ils tressaillent, se retournent et le fixent. Eben grimace un sourire, puis se rembrunit.) J'prie qu'y soye mort. (Ils le fixent. Il poursuit, terre à terre.) La soupe est prête.

- Siméon et Peter (à l'unisson): E-waye.

- Eben (regardant le ciel): L' soleil est beaw ce soir.

- Siméon et Peter (à l'unisson): E-waye. Y a d' l'or dans l'Ouest.

- Eben: E-waye. (l'index tendu) Là-bas, sur les prairies d' la colline, c'est c'que tu dis?

- Siméon et Peter (à l'unisson): En Cal'fornie!

- Eben: Ah? (il les fixe un instant avec indifférence, puis, traînant la voix:) Bôn - le souper r'froidit. (ll rentre dans la cuisine). 
- Siméon (il tressaille et clape des lèvres): J'ai faim comme chien!

- Peter (humant): Ça sent l' lard grillé!

- Siméon (ton approbateur de l'homme affamé): C'est bon, l'lard!

- Peter (même ton): L'lard, c'est l'lard.

Ils font demi-tour et s'en vont tous deux, au coude à coude, se bousculant, se frottant par moments, lourds et gauche, se hâtant vers leur nourriture comme deux bons boufs vers le repas du soir. Ils tournent le coin droit de la maison, disparaissent, et l'on peut les entendre ouvrir la porte.

\section{Scène 2}

- Cabot (relevant soudain la tête et la regardant - plein de mépris): M'connaîtras-tu jamais - toi ou quêt' aut', homme ou femme? (Secouant la tête) Non, j'calcule qu'c'était pas fait pour êt'. (Il se détourne. Abbie regarde le mur. De toute évidence incapable de garder ses pensées pour lui, il tend la main et, sans regarder sa femme, la lui pose sur le genou. Elle sursaute violemment, le regarde, voit qu'il n'a pas les yeux fixés sur elle et s'absorbe à nouveau dans la contemplation du mur, sans prêter attention à ce qu'il dit) Écoute, Abbie. Quand ça j'suis v'nu ici, c'est vieux d'près d'cinquante ans - vingt ans qu'j'avais et fort et dur comme t'en a pas vu deux - dix fois fort comme Eben et cinquante fois plus dur. Bôn - cette place ici, c'était rien qu'des champs d'pierre. Le monde y riaient quand j'l'ai prise. Y savaient pas ce que j'savais, moi. Quand tu peux faire lever du blé d'ent' les pierres, c'est qu'Djieu l'habite en toi. Pas assez forts pour ça, eux aut'! $Y$ comptaient qu'Djieu il est facile. Y riaient. Y rient pus. Y en a y sont morts par ici. $Y$ en a y sont allés dans l'Ouest, et sont morts. Y sont tous sous la terre - d'avoir été d'à suiv' un Djieu facile. Djieu, l'est pas facile. (Il secoue lentement la tête) Et j'm'ai durci. Y étaient là toujours à r'dire: «C't un homme dur», comme si c'était péché d'êt' dur, tant qu'à la fin j'leur ai r'parti: «Bôn, par l'enfer, vous allez m'tourner dur, et vous 
verrez c'que c'est!» (Brusquement) Mais la faiblesse, une fois j'ai cédé d'vant. C'était après qu'j'avais été ici deux ans. J'ai été faib' - j'm'ai dés'péré - y en avait tellement d'pierres. Des gens, toute une équipe, $y$ quittaient, $y$ cédaient, pour s'en aller dans l'Ouest. J'm'ai joint d'à eux. On a marché, marché. Trouvé des grandes prairies, des plaines d'une terre qu'était tant noire et riche comme l'or. Et pas une pierre. Et facile. Tu laboures, tu sèmes et tu restes là d'vant à fumer ta pipe en r'gardant tout pousser les cheawses. J'auraye pu êt' un homme riche - mais dans l' dedans, ça m'rev'nait, ça m'cognait - la voix d'Djieu qui m'disait «Tout ça n'veaw rien pour Moi. R'tourne à la maison!» J'ai eu peur d' cette voix, et j'ai tout laissé pour m'en r'tourner à la maison ici, et tout mon lot, toutes mes récoltes dessus, j'ai tout laissé au premier qui l' voudraye. E-waye. J'ai d' bon r'noncé à c'qu'était à moi d' droit! Djieu, l'est dur, l'est pas facile! Djieu l'est dans les pierres. Et sur cette pierre j' bâtirai Mon église - toute avec des pierres et j's'rai dans ces pierres. C'est c'qu'il a dit à Pierre. (Il soupire profondément - une pause) Les pierres. J'les ai tirées, j'les ai mises dans des murs. Tu peux lire les années d'ma vie dans ces murs, chaque jour une pierre de plus, à r'monter des collines ou r'descendre, à clore les champs qu'étaient à moi, où j'avais tout fait pousser d'rien - comme la volonté d'Djieu, comme l' serviteur d' Sa main. C'était pas facile. C'était dur et Djieu, y m'a fait dur pour ça. (Il s'arrête) Et tout l'temps toujours plus dans les solitudes. J'ai pris femme. L'a enfanté Siméon et Peter. Une bonne femme. E' travaillait dur. Vingt ans on a été mariés. E' m'a jamais connu. E' m'aidait bien mais elle a pas jamais connu à quoi faire ê' m'aidait. J'étais toujours seul. Elle est morte. Après ça, pendant un bout d' temps, f'a pas semblé tant seul. (Une pause) J'ai oublié l' compte des années. Pas d'temps à perd' à les compter. Sim et Peter, ils aidaient. La ferme, ê' s'grandissait. Et c'était tout à moi! Quand j'pensais à ça je m' sentais pas seul. (Une pause) Mais on peut pas s'atteler l'idée à la même cheawse le jour la nuit. J'ai pris une aut' femme la Mère à Eben. Elle, sa famille, y m' contestaient d'vant l'juge les actes comme quoi j'avais la ferme-ma ferme! C'est pour ça qu'Eben il est toujours à radoter son rabâchage sur la 
ferme qu'est la ferme à sa Mère. L'a enfanté Eben. L'était belle - mais douce. L'essayait bien d'ê t' dure. Pouvait pas. E' m'a jamais connu ni rien. Plus seul qu'l'enfer j'étais d'avec cette femme. Après un temps d'environ seize années, elle est morte. (Une pause) J'suis resté d'avec les garçons. Y m'détestaient d'êt' dur. J'les détestais d'êt' doux. Y convoitaient la ferme sans connaît' c' qu'elle était. Ça m'rendait amer pis qu' l'absinthe amère. Ça m' faisait peser l' âge, qu'y convoit' c'que j'avais fait pour qu'ça soye à moi. Et là c'printemps l'appel est v'nu - la voix de Djieu criant dans mon désert, du fond d'mes solitudes - va, cherche, et trouve! (Se tournant vers elle avec une étrange passion) J't'ai cherchée, j't'ai trouvée! T'es ma rose de Saron! Tes yeux sont comme... (Elle a tourné vers lui un visage inerte et le regarde avec rancune. Il la fixe un moment-puis, d'une voix dure) $\mathrm{Tu}$ t'sens plus avancée d'avoir appris tout ça que j'viens d'te dire?

\section{RÉSUMÉ - À propos d'une expérience de traduction: Désir sous les} ormes d'Eugène $O$ 'Neill. À partir d'une expérience de traduction ponctuelle (Désir sous les ormes d'Eugène O'Neill mis en scène par Matthias Langhoff en 1992), la traductrice s'efforce de rendre compte des solutions qu'elle a trouvées pour transposer l'anglo-irlandais utilisé par O'Neill comme langue de poésie. Il lui a semblé possible de traduire la dualité de cet anglais marqué par les structures du gaélique en utilisant le français marqué par les structures du breton tel qu'on l'entend encore parler en Basse Bretagne. Cependant, le recours à un parler vécu (ayant donc sa cohérence) et à une situation d'élocution semblable (de partage impossible) ont eu pour avantage, non seulement de réaliser un calque mais d'autoriser une rupture avec la norme. C'est cette rupture, acceptée en vue d'un gain d'efficacité, qui a contraint la traduction à partir des éléments ayant précisément été effacés par les autres traductions françaises de la pièce: les récurrences, le système métaphorique, la ponctuation et la graphie du texte, en tant qu'éléments constitutifs, porteurs de sens. Cet exemple très limité amène ainsi à considérer les problèmes que pose la traduction d'un sociolecte comme révélateurs d'une résistance à l'acte de traduire, inhérente à cet acte même, parce qu'issue d'une résistance à l'autre, en ce qu'il a d'étranger. 
ABSTRACT - About an Translation Experiment: Desire under the Elms by Eugene O'Neill. Starting from a particular experience (the translation of Eugene O'Neill's Desire under the Elms for a production by Matthias Langhoff in 1992), the translator attempts to convey the solutions she found to transpose the Anglo-Irish used by O'Neill as a poetic language. It seemed feasible to transpose the duality of this English sociolect, marked as it is by Gaelic structures, by using the French language, marked by Breton structures, as it is still spoken in Brittany. Use of a living language (a language therefore having its own coherence) and reference to a similar situation of impossible sharing between two languages had not only the advantage of producing a tracing of the original, but also of enabling a break with the linguistic norm. It is this break, accepted for the sake of efficiency, that made it necessary to translate the very elements that had been drawn aside by existing French translations of the play (i.e., recurrent words, the metaphoric net, punctuation and spelling) and to regard them as constituent elements that convey the meaning of the text. This very limited example consequently leads to view the problems raised by the translation of sociolects as signs of a resistance to the act of translating, inherent to the act itself. 